\title{
Improved deuteron elastic breakup energy dependence via the continuum-discretized coupled-channels method
}

\author{
M. Avrigeanu* \\ “Horia Hulubei” National Institute for Physics and Nuclear Engineering, P.O. Box MG-6, R-077125 Bucharest-Magurele, Romania
}

A. M. Moro ${ }^{\dagger}$

Departamento de FAMN, Universidad de Sevilla, Apartado 1065, E-41080 Sevilla, Spain

(Received 9 June 2010; published 9 September 2010)

\begin{abstract}
Experimental elastic-scattering angular distributions for deuteron interaction with ${ }^{63} \mathrm{Cu}$ and ${ }^{93} \mathrm{Nb}$ targets are compared with calculations performed within the continuum-discretized coupled-channels (CDCC) method, in which coupling to breakup channels is explicitly taken into account. The calculated elastic breakup cross sections are compared with the predictions of an empirical parametrization for a wide range of deuteron incident energies. The good agreement between the calculations and the systematics at the energies where data are available indicates that the CDCC method permits a useful assessment of empirical parametrizations and provides useful guidance for the extrapolation of these parametrizations beyond the energies formerly considered.
\end{abstract}

DOI: 10.1103/PhysRevC.82.037601

PACS number(s): 24.10.Eq, 24.10.Ht, 25.45.De

Introduction. The description of deuteron-nucleus interactions has been an important test for both the strength of the reaction mechanism models and the evaluation of nuclear data requested especially for fusion reactor technology. The complexity of deuteron-induced reactions that occur at low-incident energies, below the nucleon-binding energy, is the result of the high enhancement of a variety of reactions initiated by the neutrons and protons coming from the deuteron breakup [1]. However, there are already notable contributions to deuteron-induced reaction studies (e.g., Refs. [2-6]) that have taken into account only the statistical emission and eventually a "reduction factor" of the compound nucleus cross section due to "direct processes." Moreover, this reduction factor does not allow the distinction between processes such as the breakup and the stripping mechanisms that affect the different energy ranges of particles emitted through the decay of excited composite nuclei.

The difficulties in interpreting the deuteron-induced reaction data in terms of the usual reaction mechanism models have recently been investigated [1,7-12] and attempts have been made to find a consistent way to include the breakup contribution within the activation cross section calculations. Actually, the deuteron-induced reactions at low and medium energies have a great importance for the assessment of the induced radioactivity of the International Fusion Materials Irradiation Facility (IFMIF) components. Moreover, recent calculations of deuteron activation have shown that deuterons are more important than neutrons, due to a value of about 70 for the ratio of the deuteron- and neutron-induced activity, respectively [13].

The physical picture of the deuteron breakup in the Coulomb and nuclear fields of the target nucleus considers two distinct processes, namely, the elastic breakup (EB), in which the target nucleus remains in its ground state and

\footnotetext{
*mavrig@ifin.nipne.ro

†moro@us.es
}

none of the deuteron constituents interacts with it, and the inelastic breakup or breakup fusion (BF), where one of these deuteron constituents interacts with the target nucleus while the remaining one is detected. Despite the important theoretical studies devoted to the breakup processes (e.g., see review papers Refs. [14-16] and references therein), only the elastic breakup contribution can be accurately calculated so far within the continuum-discretized coupled-channels (CDCC) method [17-23]. Several attempts have been proposed in the literature to calculate the inelastic breakup component from the distorted-wave Born approximation (DWBA) [24-26] or CDCC methods [27,28]. However, there is no clear consensus among these works and a systematic assessment of the validity of these approaches against existing data remains to be done.

Thus, the total and inelastic breakup components are typically estimated empirically [7,10]. For example, Kalbach Walker [7] gives parametrizations for the total proton- and neutron-emission breakup cross sections $\left(\sigma_{\mathrm{BU}}^{p}\right.$ and $\left.\sigma_{\mathrm{BU}}^{n}\right)$, while a second parametrization [10] considers equal breakup contributions for proton and neutron emission but supplementarily gives all the breakup components, that is, the total $\left(\sigma_{\mathrm{BU}}^{p / n}\right)$, elastic $\left(\sigma_{\mathrm{EB}}\right)$, and inelastic $\left(\sigma_{\mathrm{BF}}^{p / n}\right)$ breakup cross sections.

The empirical parametrization of the total proton-emission breakup fraction, $\sigma_{\mathrm{BU}}^{p} / \sigma_{R}$, where $\sigma_{R}$ is the deuteron reaction cross section and $\sigma_{\mathrm{BU}}^{p}=\sigma_{\mathrm{BF}}^{p}+\sigma_{\mathrm{EB}}$, has been obtained [10] on the basis of experimental systematics [29-33]. Thus, protonemission spectra and angular distributions from deuteroninduced reactions on nuclei from $\mathrm{Al}$ to $\mathrm{Pb}$ at incident energies from 15 to $80 \mathrm{MeV}$ have been concerned in this respect. However, an energy range of only $15-30 \mathrm{MeV}$ has been available for the empirical elastic breakup fraction $\sigma_{\mathrm{EB}} / \sigma_{R}$ systematics [31,33]. Given the interest in the deuteron-activation cross sections for incident energies up to $60 \mathrm{MeV}$, the extension of the elastic breakup parametrization [10] at higher incident energies should be checked. In Fig. 1 the energy dependence of the total, elastic, and inelastic proton-emission breakup cross 


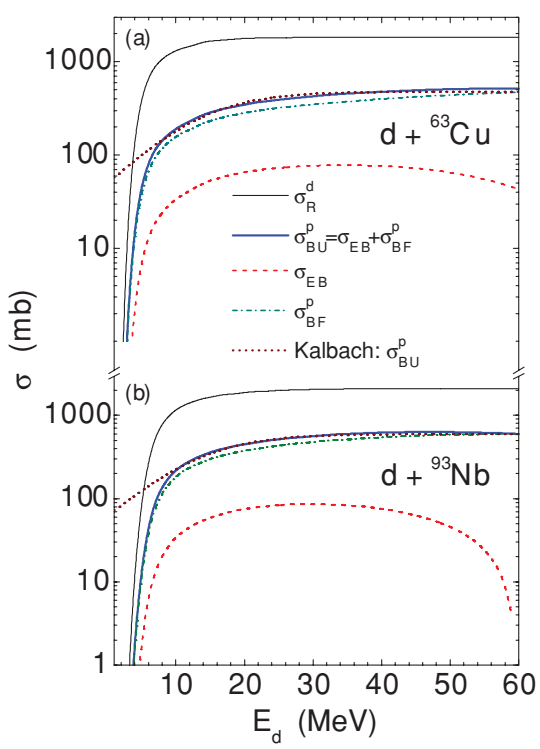

FIG. 1. (Color online) Energy dependence of the total (thick solid curves), elastic (dashed curves), and inelastic (dot-dashed curves) proton-emission breakup cross sections given by Ref. [10] and of the total proton-emission breakup cross sections [7] (dotted curves) for the deuteron interactions with (a) ${ }^{63} \mathrm{Cu}$ and (b) ${ }^{93} \mathrm{Nb}$. The corresponding total reaction cross section is shown by the thin curves.

sections following Ref. [10] is shown as well as the total proton-emission breakup cross sections [7] for the deuteron interactions with the target nuclei ${ }^{63} \mathrm{Cu}$ and ${ }^{93} \mathrm{Nb}$. It results that the predictions for the total proton-emission breakup cross sections given by both parametrizations are close for deuteron incident energies above $\sim 8 \mathrm{MeV}$, while at the lowest energies the total proton-emission breakup cross section provided by the latter parametrization [7] become even higher than the deuteron total reaction cross section. However, the elastic breakup cross sections given by the empirical parametrization [10] decrease with the incident energy beyond the energy range within which it was established. This trend is thus opposite to that of the total breakup cross section. However, in the absence of any available experimental deuteron elastic breakup cross section at incident energies above $30 \mathrm{MeV}$, the correctness of an eventual parametrization extrapolation may be checked by a comparison of its predictions and the CDCC method $[15,16]$ results.

CDCC calculations. A detailed description of the CDCC formalism is given elsewhere [16,17,19-22], and hence only a brief description of the method is given here.

In the CDCC formalism, the $d+$ target scattering process is treated within a three-body model, comprising the two-body projectile and the target. This three-body system is described by the model Hamiltonian [16]

$$
H=K_{r}+K_{R}+V_{n p}(\mathbf{r})+U_{n}(\mathbf{R}-\mathbf{r} / 2)+U_{p}(\mathbf{R}+\mathbf{r} / 2),
$$

where $V_{n p}$ is the interaction between $n$ and $p$, the vector $\mathbf{r}$ is the proton-neutron relative coordinate, $\mathbf{R}$ is the coordinate of the center of mass of the $p-n$ pair relative to the target nucleus, and $U_{p}$ and $U_{n}$ are, respectively, the proton-target and neutron-target interactions, including the Coulomb potential in the former. The operators $K_{r}$ and $K_{R}$ are the kinetic energies associated with $\mathbf{r}$ and $\mathbf{R}$.

The breakup component is treated as an inelastic excitation of the projectile due to the nuclear and Coulomb interactions with the target. This inelastic excitation is included through the coupling of the projectile unbound excited states in the solution of the scattering problem by means of the coupledchannels approach. Because the deuteron has no bound excited states, any excitation in the $p-n$ coordinate will break it up into a proton and a neutron. To deal with a finite set of coupled equations, an essential feature of the CDCC method is the introduction of a discretization procedure, in which the continuum spectrum is represented by a finite and discrete set of square-integrable functions. The most widely used method of continuum discretization is the so-called binning method $[15,16]$, in which the continuum spectrum is truncated at a maximum excitation energy $\left(E_{\max }^{*}\right)$ and divided into a set of energy (or relative momentum) intervals. Each interval, or bin, is represented by a single square-integrable function, calculated by averaging the scattering states for the $p$ - $n$ relative motion within the bin width. The use of squareintegrable functions, although not essential, is convenient to guarantee that continuum-continuum couplings are well behaved.

Moreover, the $p-n$ relative angular momentum is also restricted by considering only a limited number of partial waves in order to deal with a finite set of coupled equations. Finally, the three-body scattering wave function is expanded over the internal states of the deuteron as follows:

$$
|\Psi(E)\rangle=\sum_{i=0}^{N}\left|\phi_{i}, \chi_{i}\right\rangle,
$$

where $\left|\phi_{0}\right\rangle$ is the ground-state wave function and $\phi_{i}(i \neq 0)$ are the averaged (within each bin) continuum wave functions. The radial functions $\chi_{i}(\mathbf{R})$ describe the projectile-target relative motion for the elastic $(i=0)$ and breakup $(i \neq 0)$ components.

This procedure should be regarded as a practical method of making the problem numerically solvable, rather than an additional approximation. In practical calculations, one has to study the convergence with respect to the maximum excitation energy, the number of bins, and the number of partial waves included for the internal motion. In addition, it has been shown that the calculated observables are essentially independent of the discretization procedure [17,19,21].

The neutron-proton bound and continuum states are modeled with a simple Gaussian interaction fitted to the deuteron binding energy [15] and ${ }^{3} S_{1}$ phase shifts:

$$
V_{n p}(r)=-V_{0} e^{-\left(r / r_{0}\right)^{2}},
$$

where $V_{0}=72.15 \mathrm{MeV}$ and $r_{0}=1.484 \mathrm{fm}$. Continuum states with orbital angular momentum $\ell=0,1$, and 2 for the $p-n$ relative motion were considered. The proton and neutron intrinsic spins were ignored for simplicity. The calculations were performed with the coupled-channels code FRESCO [34]. 
Discussion. In the standard formulation of the CDCC method, the three-body model space considers only the target ground state and hence the method provides only the elastic breakup and leaves out the inelastic breakup processes [19]. However, in order to take into account the effect of the excluded channels, the proton- and neutron-target interactions $U_{p}(\mathbf{R}+\mathbf{r} / 2)$ and $U_{n}(\mathbf{R}-\mathbf{r} / 2)$ are usually taken as the central nuclear part of the proton and neutron phenomenological optical model potentials (OMPs) at half the deuteron incident energy, $E_{d} / 2$. The neutron and proton global OMPs of Koning and Delaroche [35] have been used in the present calculations. For deuteron energies below $\sim 30 \mathrm{MeV}$, the geometry parameters of the nucleon real and surface imaginary potentials have been adjusted to obtain a suitable description of the measured deuteron elastic-scattering angular distributions. The experimental elastic-scattering angular distributions for deuteron interaction with ${ }^{63} \mathrm{Cu}$ and ${ }^{93} \mathrm{Nb}$ target nuclei $[36,37]$ are compared with the present CDCC calculations in Figs. 2(a)-2(c) and 3(a)-3(d). Their rather good agreement supports the reliability of the OMPs used in the CDCC calculations.

Finally, the energy dependence of the elastic breakup cross sections, provided by the excitation of the continuum spectrum, for the deuteron interaction with ${ }^{63} \mathrm{Cu}$ and ${ }^{93} \mathrm{Nb}$, is compared with the prediction of empirical systematics [10] in Figs. 2(d) and 3(e), respectively. The elastic breakup cross sections corresponding to the Kleinfeller et al. systematics (Table III of Ref. [31]) are also shown. The agreement of the
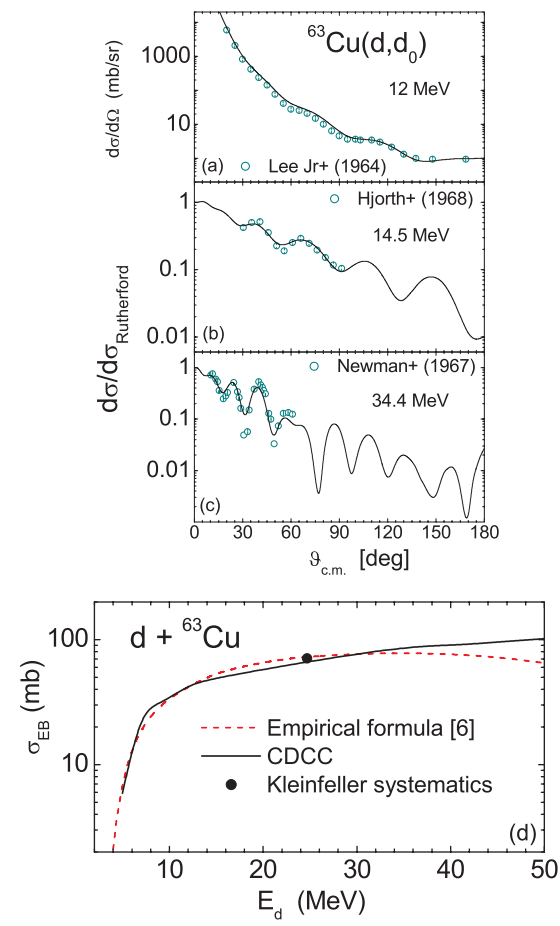

FIG. 2. (Color online) (a)-(c) Comparison of measured [36] and calculated (CDCC) angular distributions of deuteron elastic scattering on ${ }^{63} \mathrm{Cu}$ at $E_{d}=12,14.5$, and $34.4 \mathrm{MeV}$. (d) Energy dependence of the empirical [10] (dashed curve) and CDCC (solid line) elastic breakup cross sections for deuteron scattering with ${ }^{63} \mathrm{Cu}$. The solid circle is the value from Kleinfeller systematics [31].
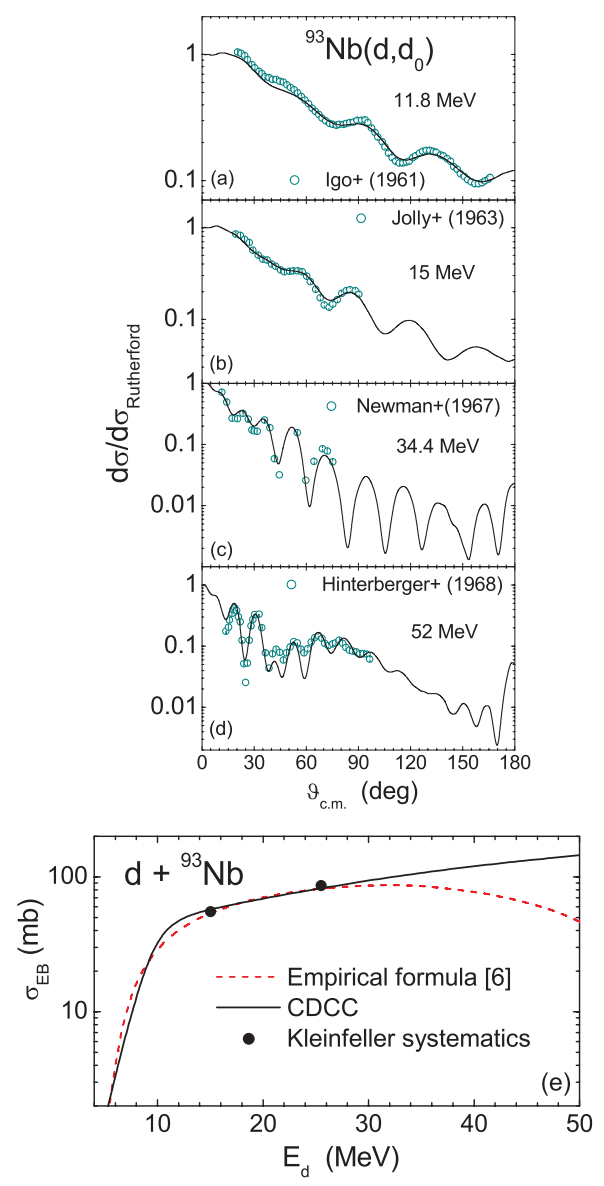

FIG. 3. (Color online) (a)-(d) Comparison of measured [37] and calculated (CDCC) angular distributions of deuteron elastic scattering on ${ }^{93} \mathrm{Nb}$ at $E_{d}=11.8,15,34.4$, and $52 \mathrm{MeV}$. (e) The same as in Fig. 2(d) but for deuteron scattering on ${ }^{93} \mathrm{Nb}$.

CDCC elastic breakup cross sections with this systematics can be considered as the validation of the present approach for the former. Moreover, the comparison shown in Figs. 2(d) and 3(e) points out that the CDCC calculations lead to elastic breakup cross sections that follow the total breakup cross-section behavior as well as that of the reaction cross section shown formerly in Figs. 1(a) and 1(b). Therefore the present analysis makes clear that the extension of the empirical parametrization of the elastic breakup cross sections beyond the energies considered in this respect should be done with caution. The CDCC method provides thus a first useful guidance for the assessment of the accuracy of these extrapolations and may help to improve existing parametrizations. Moreover, additional experimental data concerning deuteron-activation cross sections, inclusive proton and neutron spectra, and deuteron elastic-scattering angular distributions are needed to validate the theoretical calculations over enlarged energy and target mass domains.

Acknowledgments. This work was partly supported by CNCSIS Project PNII-IDEI-43/2008, Spanish Ministerio de Ciencia e Innovación Project FPA2009-07653, and Spanish Conso-lider-Ingenio 2010 Programme CPAN (CSD200700042). 
[1] P. Bém, E. Šimečková, M. Honusek, U. Fischer, S. P. Simakov, R. A. Forrest, M. Avrigeanu, A. C. Obreja, F. L. Roman, and V. Avrigeanu, Phys. Rev. C 79, 044610 (2009).

[2] S. Sudar and S. M. Qaim, Phys. Rev. C 50, 2408 (1994); 53, 2885 (1996).

[3] S. I. Al Quraishi, C. E. Brient, S. M. Grimes, T. N. Massey, J. Oldendick, and R. Wheeler, Phys. Rev. C 62, 044616 (2000).

[4] A. V. Voinov et al., Phys. Rev. C 74, 014314 (2006).

[5] A. V. Voinov, S. M. Grimes, C. R. Brune, M. J. Hornish, T. N. Massey, and A. Salas, Phys. Rev. C 76, 044602 (2007).

[6] A. V. Voinov, B. M. Oginni, S. M. Grimes, C. R. Brune, M. Guttormsen, A. C. Larsen, T. N. Massey, A. Schiller, and S. Siem, Phys. Rev. C 79, 031301(R) (2009).

[7] C. Kalbach Walker, TUNL Prog. Rep. XLII, 2002-2003, pp. 80-83 [www.tunl.duke.edu/publications/tunlprogress/2003/].

[8] M. Avrigeanu, W. von Oertzen, U. Fischer, and V. Avrigeanu, Nucl. Phys. A 759, 327 (2005).

[9] M. Avrigeanu, W. von Oertzen, H. Leeb, F. L. Roman, and V. Avrigeanu, in Proceedings of the 11th International Conference on Nuclear Reaction Mechanisms, June 2006, Varenna, Italy, edited by E. Gadioli (Ricerca Scientifica ed Educazione Permanente, Milan, 2006), p. 123.

[10] M. Avrigeanu, W. von Oertzen, R. A. Forrest, A. C. Obreja, F. L. Roman, and V. Avrigeanu, Fusion Eng. Des. 84, 418 (2009).

[11] M. Avrigeanu and V. Avrigeanu, EPJ Web Conf. 2, 01004 (2010).

[12] M. Avrigeanu and V. Avrigeanu, J. Phys. Conf. Ser. 205, 012014 (2010).

[13] R. A. Forrest and I. Cook, Fusion Eng. Des. 82, 2478 (2007).

[14] G. Baur, F. Rosel, D. Trautmann, and R. Shyam, Phys. Rep. 111, 333 (1984).

[15] M. Kamimura, M. Yahiro, Y. Iseri, Y. Sakuragi, H. Kameyama, and M. Kawai, Prog. Theor. Phys. 89, S1 (1986).

[16] N. Austern, Y. Iseri, M. Kamimura, M. Kawai, G. Rawitscher, and M. Yahiro, Phys. Rep. 154, 125 (1987).

[17] R. A. D. Piyadasa, M. Kawai, M. Kamimura, and M. Yahiro, Phys. Rev. C 60, 044611 (1999).

[18] J. A. Tostevin, F. M. Nunes, and I. J. Thompson, Phys. Rev. C 63, 024617 (2001).

[19] A. M. Moro and F. M. Nunes, Nucl. Phys. A 767, 138 (2006).
[20] A. Deltuva, A. M. Moro, E. Cravo, F. M. Nunes, and A. C. Fonseca, Phys. Rev. C 76, 064602 (2007).

[21] A. M. Moro, J. M. Arias, J. Gómez-Camacho, and F. PérezBernal, Phys. Rev. C 80, 054605 (2009).

[22] A. M. Moro, F. M. Nunes, and R. C. Johnson, Phys. Rev. C 80, 064606 (2009).

[23] T. Ye, Y. Watanabe, K. Ogata, and S. Chiba, Phys. Rev. C 78, 024611 (2008); T. Ye, Y. Watanabe, and K. Ogata, ibid. 80, 014604 (2009).

[24] T. Udagawa and T. Tamura, Phys. Rev. C 33, 494 (1986).

[25] M. Ichimura, N. Austern, and C. M. Vincent, Phys. Rev. C 32, 431 (1985).

[26] M. S. Hussein and K. W. McVoy, Nucl. Phys. A 445, 124 (1985).

[27] A. Diaz-Torres and I. J. Thompson, Phys. Rev. C 65, 024606 (2002).

[28] S. Hashimoto, K. Ogata, S. Chiba, and M. Yahiro, Prog. Theor. Phys. 122, 1291 (2009).

[29] J. Pampus, J. Bisplinghoff, J. Ernst, T. Mayer-Kuckuk, J. Rama Rao, G. Baur, F. Rosel, and D. Trautmann, Nucl. Phys. A 311, 141 (1978).

[30] J. R. Wu, C. C. Chang, and H. D. Holmgren, Phys. Rev. C 19, 370 (1979).

[31] J. Kleinfeller, J. Bisplinghoff, J. Ernst, T. Mayer-Kuckuk, G. Baur, B. Hoffmann, R. Shyam, F. Rosel, and D. Trautmann, Nucl. Phys. A 370, 205 (1981).

[32] N. Matsuoka, M. Kondo, A. Shimizu, T. Saito, S. Nagamachi, H. Sakaguchi, A. Goto, and F. Ohtani, Nucl. Phys. A 345, 1 (1980).

[33] M. G. Mustafa, T. Tamura, and T. Udagawa, Phys. Rev. C 35, 2077 (1987)

[34] I. J. Thompson, Comput. Phys. Rep. 7, 167 (1988); Version FRES 2.3, May 2007.

[35] A. J. Koning and J. P. Delaroche, Nucl. Phys. A 713, 231 (2003).

[36] L. L. Lee Jr. and J. P. Schiffer, Phys. Rev. 134, B765 (1964); S. A. Hjorth and E. K. Lin, Nucl. Phys. A 116, 1 (1968); E. Newman, L. C. Becker, and B. M. Freedom, ibid. 100, 225 (1967).

[37] R. K. Jolly, E. K. Lin, and B. L. Cohen, Phys. Rev. 130, 2391 (1963); F. Hinterberger, G. Mairle, U. Schmidt-Rohr, G. J. Wagner, and P. Turek, Nucl. Phys. A 111, 265 (1968); G. Igo, W. Lorenz, and U. Schmidt-Rohr, Phys. Rev. 124, 832 (1961). 